\title{
Cardio-Oncology: A Tale of Two Diseases
}

Ganatra $\mathbf{S}^{1,2}$

${ }^{1}$ Department of Cardiovascular Medicine, Lahey Hospital \& Medical Center, Burlington, Massachusetts, USA

${ }^{2}$ Cancer Survivorship Program, Lahey Hospital and Medical Center, Burlington, Massachusetts, USA

*Corresponding author: Sarju Ganatra, Department of Cardiovascular Medicine, Lahey Hospital \& Medical Center, 41 Mall Road, Burlington, MA 01805, USA, Tel: + 781-744-8460; Fax: 781-744-5261; E-mail: sarju.ganatra@lahey.org

Received date: November 10, 2017; Accepted date: November 17, 2017; Published date: November 24, 2017

Copyright: () 2017 Ganatra S. This is an open-access article distributed under the terms of the Creative Commons Attribution License, which permits unrestricted use, distribution, and reproduction in any medium, provided the original author and source are credited.

\section{Editorial}

Cancer and cardiovascular disease (CVD) are one of the fastest growing and most prevalent diseases in the United States, in turn, greater overlap is expected. They both are complex entities and together they represent two most common causes of morbidity and mortality in developed countries. Nearly 14.5 million Americans with a history of cancer were alive in 2014 and by 2024 that number will increase to nearly 19 million [1]. Evidence increasingly shows that cancer and CVD are interlinked through common risk factors, cooccurrence in an aging population, and through the deleterious effects of cancer treatment on cardiovascular health. Great progress has been made in prevention, early diagnosis and treatment of these diseases; yet both remain extraordinary concerns both from public health perspective and with regard to devastation and suffering that those afflicted with a dual diagnosis. Increases in cardiovascular morbidity and mortality now threaten to offset some of the advancements in cancer-related survival.

The relationship between cancer and CVD is bidirectional. Well known risk factors for CVD such as tobacco, obesity, physical inactivity, poor nutrition, diabetes, alcohol, hypertension and hyperlipidemia are also risk factors for cancer. Not only that, but an individual cardiovascular risk factors like hypertension in a cancer survivor appear to carry greater risk than hypertension in an individual without a history of cancer. Additionally, each additional risk factor found in a cancer survivor appears to increase CVD risk to a greater magnitude than a simple additive effect [2]. On other hand, cancer survivors are more likely to have traditional cardiovascular risk factors compared with age-matched controls [3,4]. The extensive overlap in risk factors between these seemingly diverse diseases suggests some common basic molecular pathways or networks. Chronic inflammation may have a considerable role because it contributes to both diseases and occurs in conditions such as obesity, diabetes mellitus, hypertension, and dyslipidemia.

Cardiotoxicity is a well-established complication of oncology therapies. Chemotherapeutic agents can cause a wide spectrum of short- and long-term cardiotoxic effects. Cardiomyopathy resulting from anthracyclines (AC) is a classic example. Since the successful introduction in the early 1950s, anthracyclines continue to be the most commonly used chemotherapeutic agents to treat a variety of solid and hematologic tumors in both adults and children. However, due to a high incidence of cardiotoxicity, their utility is limited in patients with a pre-existing cardiomyopathy or heart failure. Several strategies have been evaluated for prevention of cardiotoxicity including close monitoring with strain echocardiography and cardiac biomarkers as well as prophylactic use of angiotensin-converting enzyme inhibitor and/or $\beta$-blocker for neurohormonal blockade in patients with normal systolic function. However, the evidence is not very strong and there is no consensus on this issue among experts. These strategies fail to address the challenge faced by patients with pre-existing cardiomyopathy as they are often excluded from receiving potentially lifesaving/sustaining anthracycline based treatment with fear of further cardiotoxicity and development of heart failure. Dexrazoxane (DEX), an iron chelator, protects against the cardiotoxic effects of AC but is underutilized given its narrow FDA approval for adults with advanced breast cancer with a cumulative treatment dose of at least $300 \mathrm{mg} / \mathrm{m} 2$ and concern for reduced anti-tumor response rates. Although, the cumulative dose of $\mathrm{AC}$ is an independent predictor of cardiotoxicity, there is no minimum safe dose and patients with preexisting cardiomyopathy are at higher risk of future cardiotoxicity [5]. Although there was initial concern regarding the reduced anti-cancer effects of AC when used with DEX, this finding has not been reproduced in multiple clinical studies. In contrast, in pediatric population with hematological malignancies, it has been used successfully and has shown protective effect against AC related cardiotoxicity when used concomitantly from the beginning of the AC therapy [6]. The role of DEX in adult non-breast cancer patients with preexisting systolic dysfunction has been evaluated and has shown promising results and its should be studied prospectively in this population [7]

Although, cardiomyopathy is a prototypical cardiotoxicity, chemotherapeutic agents also causes brady and tachyarrhythmias, hypertension, arterial and venous thrombosis, coronary artery spasm, atherosclerosis, valvular heart disease, myocarditis and so on. In the past decade, an explosion of novel cancer therapies often targeted and more specific than conventional therapies, has revolutionized oncology therapy and dramatically changed cancer prognosis. However, some of these therapies have introduced an assortment of cardiovascular complications. For example, Ibrutinib, which is a novel Bruton's tyrosine kinase inhibitor approved for treatment of B-cell malignancies is associated with up to 10 -fold increased incidence of AF as well as increased risk of bleeding. In addition, several major interactions with drugs commonly used to manage AF complicate the management of these patients $[8,9]$. Also, it is interesting to note that new onset AF after diagnosis of malignancy is associated with significantly higher risk of thromboembolism and heart failure even after adjusting for known risk factors. Immune checkpoint inhibitors have transformed the treatment of several cancers by releasing restrained antitumor immune response, but immune-related adverse events can occur. Although the incidence of myocarditis is relatively low, most reported cases have been fulminant.

Radiation therapy (RT) was and still remains very effective treatment for several malignancies such as lung, thyroid, breast, Hodgkin's lymphoma just to name few. Unfortunately mediastinal 
radiotherapy is associated with a 7 fold increased risk of coronary artery disease [10]. Valvular heart disease, pericardial calcification and cardiomyopathy are also very well-known complications of mediastinal RT. The challenge here is multifold as there is no surveillance guidelines, these complications occur many years after the initial therapy, by the time many of the patients are not under surveillance and also the treatment is extremely challenging in these patients.

Cancer patients are considered to have high bleeding risk but at the same time, it is a pro-thrombotic state. This issue poses a unique challenge and balance decision is required when it comes to antiplatelet therapy after percutaneous coronary intervention. Most experts prefer shorter duration of dual antiplatelet therapy (DAPT) out of concern for bleeding and with expectant cancer related procedure. With this approach, bare-metal stent (BMS) has been preferred by most. However, the current evidence with newer generation stent technology demonstrates the feasibility of shorter duration of DAPT, without increasing the risk of stent thrombosis and bleeding, while maintaining improved efficacy compared with BMS. Considering the developing evidence thus far, it is time to rethink the choice of stent in cardio-oncology $[11,12]$.

Almost all of the approved antineoplastics have been shown to induce some type of cardiotoxicity. However, concerns about cardiotoxicity from antitumor drugs should be weighed against survival or curability benefits. In 2015 , oncology was a natural choice as the initial focus of the U.S. government Precision Medicine Initiative. In 2016, cancer moonshot was announced to accelerate cancer research and to make more therapies available. However, cancer therapy related cardiotoxicity has not been given deserved attention. Even if we are successful in treating or curing many cancers, but if we fail to prevent severe cardiotoxicity, the moonshot can fall short in achieving the desired outcomes. Integration of precision medicine tools such as human pluripotent stem cell-derived cardiomyocytes (PSC-CMs) in vitro model and pharmacogenomic testing in development of new cancer therapies can improve cardiotoxicity assessment in preclinical studies and can also help developing the prevention, monitoring as well as treatment strategies for each individual [13].

Cardiovascular disease in patients with cancer is complex, and treatment needs to be individualized. Although the field of cardiooncology was born long time ago with first recognition of cardiotoxicity due to anthracyclines in 1960s, it has emerged only in recent years as a formal subspecialty. The scope of cardio-oncology includes not only the prevention, detection, monitoring and treatment of cardiovascular toxicity related to cancer therapy but also to guide the cancer therapy development with minimal cardiovascular toxicity. The goal is to provide optimal care for patients with cancer and cardiovascular disease from cancer diagnosis into survivorship.

\section{References}

1. DeSantis CE, Lin CC, Mariotto AB, Seigel RL, Stein KD, et al. (2014) Cancer treatment and survivorship statistics 2014. CA Cancer J Clin 64: 252-271.

2. Armstrong GT, Oeffinger KC, Chen Y, Kawashima T, Yasui Y, et al. (2013) Modifiable risk factors and major cardiac events among adult survivors of childhood cancer. J Clin Oncol 31: 3673-3680.

3. Oeffinger KC, Mertens AC, Sklar CA, Kawashima T, Hudson MM, et al. (2006) Chronic health conditions in adult survivors of childhood cancer. N Engl J Med 355: 1572-1582.

4. Armenian SH, Xu L, Ky B, Sun C, Farol LT, et al. (2016) Cardiovascular Disease Among Survivors of Adult-Onset Cancer: A Community-Based Retrospective Cohort Study. J Clin Oncol 34: 1122-1130.

5. Cardinale D, Colombo A, Bacchiani G, Tedeschi I, Meroni CA, et al. (2015) Early Detection of Anthracycline Cardiotoxicity and Improvement With Heart Failure Therapy. Circulation 131: 1981-1988.

6. Lipshultz SE, Rifai N, Dalton VM, Levy DE, Silverman LB, et al. (2004) The Effect of Dexrazoxane In Myocardial Injury in Doxorubicin-treated Children with Acute Lymphoblastic Leukemia. N Engl J Med 351: 145-153.

7. Ganatra S, Majithia A, Shah S (2017) Expanding The Role of Dexrazoxane For Cardioprotection: Use in a Patient with Preexisting Systolic Dysfunction and No Prior Anthracycline Exposure. J American College of Cardiol 69: p2389.

8. Ganatra S, Majithia A, Shah S (2017) Challenges in Ibrutinib Associated Atrial Fibrillation. J American College Cardiol 69: p2308.

9. Ganatra S, Sharma A, Majithia A, Shah S (2017) Management Strategies for Ibrutinib-Associated AF.

10. Aleman BMP, Belt-Dusebout AWVD, Bruin MLD, Veer MBVt, Baaijens MHA, et al. (1886) Late cardiotoxicity after treatment for Hodgkin lymphoma. Blood 109: 1878-1886.

11. Ganatra S, Sharma A, Levy MS (2017) Re-Evaluating the Safety of DrugEluting Stents in Cancer Patients. J Am Coll Cardiol Intv 10: 2334-2337.

12. Ganatra S, Sharma A, Levy MS (2017) A differing opinion on primary percutaneous coronary intervention in patients who have had cancer: stent choice in onco-cardiology revisited. Mayo Clin Proc 92: 1315-1316.

13. Sheng CC, Amiri KL, Palmby T, Force T, Hong CC, et al. (2016) JACC: Basic to Translational Science. JACC 1: 386-398. 